\title{
A New Approach to Modeling and Controlling a Pneumatic Muscle Actuator-Driven Setup Using Back Propagation Neural Networks
}

\author{
Jun Zhong $\mathbb{D}^{1,2}$ Xu Zhou, ${ }^{2,3}$ and Minzhou Luo ${ }^{1,2}$ \\ ${ }^{1}$ College of Mechanical \& Electrical Engineering, Hohai University, Changzhou, 213022 Jiangsu Province, China \\ ${ }^{2}$ Jiangsu Key Laboratory of Special Robot Technology, Hohai University, Changzhou, 213022 Jiangsu Province, China \\ ${ }^{3}$ College of Internet of Things Engineering, Hohai University, Changzhou, 213022 Jiangsu Province, China
}

Correspondence should be addressed to Jun Zhong; zhongjun@hhu.edu.cn

Received 3 May 2018; Accepted 27 August 2018; Published 14 October 2018

Academic Editor: Junpei Zhong

Copyright (c) 2018 Jun Zhong et al. This is an open access article distributed under the Creative Commons Attribution License, which permits unrestricted use, distribution, and reproduction in any medium, provided the original work is properly cited.

Pneumatic muscle actuators (PMAs) own excellent compliance and a high power-to-weight ratio and have been widely used in bionic robots and rehabilitated robots. However, the high nonlinear characteristics of PMAs due to inherent construction and pneumatic driving principle bring great challenges in applications acquired accurately modeling and controlling. To tackle the tricky problem, a single PMA mass setup is constructed, and a back propagation neural network (BPNN) is employed to identify the dynamics of the setup. An offline model is built up using sampled data, and online modifications are performed to further improve the quality of the model. An adaptive controller based on BPNN is designed using gradient descent information of the built-up model. Experiments of identifying the PMA setup using BPNN and position tracking by adaptive BPNN controller are performed, and results demonstrate the good capacity in accurate controlling of the PMA setup.

\section{Introduction}

Pneumatic muscle actuators (PMAs) show great potential in bionic robots $[1,2]$, rehabilitation devices $[3,4]$, and industrial applications $[5,6]$ due to the characteristics of lightweight and compliance. PMA mainly consists of three parts: rubber bladder, braided fabric, and end caps with an air inlet. A braided fabric is mixed with a rubber bladder and produces large contractile force when the rubber bladder is inflated by compressed air. As we all know, PMA is a highly nonlinear and time-varying system due to its inherent construction and pneumatic driving way. The existed high nonlinearities and hysteresis make it difficult to realize a precise control of PMA, and researchers have paid great attention to this tricky problem [7-19]. Chang designed a 2-DOF rehabilitation robot actuated by pneumatic muscle actuators and proposed an adaptive self-organizing fuzzy sliding mode controller for the robot [12]. In the controller, the fuzzy sliding surface helped to reduce the number of fuzzy rules whilst self-organizing learning mechanism regulates fuzzy rules online. Amar et al. proposed a robust controller integrated with RBFNN and an interval type-2 fuzzy logic to avoid difficult modeling and handling of the uncertainties of the artificial muscle-driven 2-DOF robot manipulator [13]. Andrikopoulos et al. designed an advanced nonlinear PID controller for pneumatic muscle actuators [14]. Wu et al. designed a phenomenological PMA model consisting of a contractile element, spring element, and damping element in parallel and proposed a novel nonlinear disturbance observer-based dynamic surface control for position tracking of a PMA system [15]. Jouppila et al. built up a single-input single-output nonlinear model for a PMA and designed a sliding mode control strategy to control a PWM-driven PMA system [16]. Lin et al. investigated pressure-length hysteresis characteristics using a Prandtl-Ishlinskii (P-I) model and designed different feedback control strategies with a P-I model as feed-word hysteresis compensation [17]. Andrikopoulos et al. established a piecewise affine system model to approximate a PMA and designed a switching model predictive position controller scheme [18]. Ba et al. derived a grey 


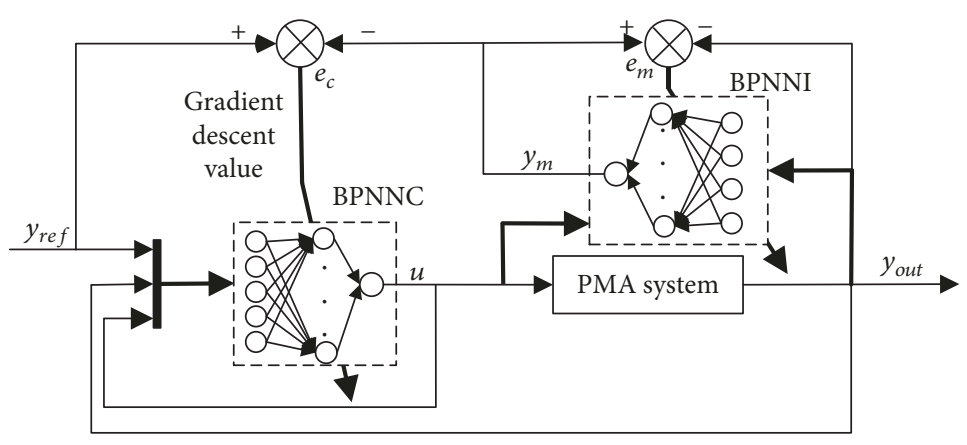

FIgURe 1: Adaptive neural network controlling scheme of PMA system.

box model of pneumatic muscle actuator using a neural network and an advanced controller integrated of designed networks, sliding mode, and backstepping techniques [19].

Back propagation neural networks (BPNNs) have a great capacity in model approximation and adaptive control due to superior nonlinear mapping ability and a flexible network structure and are widely used in robotics [20], industrial applications [21], and medical apparatus and instruments [22]. Tu et al. modeled the static friction in a robot joint by using a BP neural network to replace traditional methods [23]. Cai et al. adopted BP neural networks to identify inverse kinematics of a spherical robot [24]. Yang et al. used a neural network approximation technique to compensate the unknown dynamics of both the robot arms and the manipulated object [25].

From the analysis mentioned above, we find that different models of PMAs have been proposed, and some advanced controlling strategies were applied to PMAs. However, neural network adaptive controlling schemes combined with neural network modeling method have not been applied to PMAs. This paper adopts BPNN to approximate the dynamic behaviors of the PMA system and devises an adaptive BPNN controller to improve position controlling accuracy of PMA. The paper is organized as follows: In Section 2, a controlling scheme of the PMA system is proposed, and a BPNN identifier and an adaptive BP neural network controller of PMA system are designed, respectively. Section 3 realizes the approximation of the dynamic behaviors of a single PMA mass system using BPNN and performs trajectories tracking experiments by integration of BPNN identifier and BPNN controller. Conclusion is made in Section 4.

\section{BP Neural Network Identifier and Adaptive BP Neural Network Controller of PMA System}

2.1. Controlling Scheme of PMA System. Considering that PMA is a high nonlinear system, a neural network identifier (NNI) is employed to approximate its complex dynamics. Based on the designed NNI, a neural network controller (NNC) tackles the tracking challenges of referred trajectories applied to the PMA system. The pack propagation neural network (BPNN) owns strong nonlinear mapping capacity and flexible network structure and has been widely used in system modeling, intelligent control, information process, and pattern recognition. This paper employs the BPNN to realize dynamic modeling and adaptive position control of PMA.

The designed overall architectural structure of the controlling scheme is presented in Figure 1. Referred signal is $y_{\text {ref }}$, the actual output of PMA system is $y_{\text {out }}$, and the output of BPNNI is $y_{m}$. The output of BPNNC, i.e., $u$, is the controlling voltage of proportional valve. Structural parameters of back propagation neural network identifier (BPNNI) are tuned offline firstly, and optimal initial values are acquired to avoid large vibration at the beginning of actual application. Considering the inherent nonlinearities, time-varying parameters, and high sensitivity to the payload of the PMA mechanism, parameters of BPNNI are continuously tuned online to achieve better approximation of dynamics of PMA. The function of BPNNI is to provide a back propagation neural network controller (BPNNC) of gradient descent information.

BPNNC is presented to adaptively deal with high nonlinearities and improve trajectory tracking accuracy of PMA. Structural parameters of BPNNC is tuned online according to gradient information from BPNNI. The criteria of tuning BPNNC is minimizing $e_{c}=y_{\text {ref }}-y_{m}$. By regulating BPNNC, a more accurate signal $u$ can be produced and transient tracking errors can be decreased faster.

\subsection{BP Neural Network Identifier of PMA System}

2.2.1. Basic Structure. The inherent structure of PMA and pneumatic driving mode make PMA highly nonlinear and pose great challenges to model system dynamics accurately. Vo-Minh et al. pointed out the nonlocal memory behavior of PMA [26]. Hence, the nonlinear dynamics of the PMA system is described by a complex unknown function $f$ as follows:

$$
y(k+1)=f[y(k), \cdots, y(k-n), u(k+1), u(k), \cdots, u(k-m)],
$$

where $y(k+1)$ is the displacement of PMA at $(k+1)$ th instant, $y(k-n)$ is the displacement at $(k-n)$ th instant, and $u(k+1)$ is the input voltage of the proportional valve at $(k+1)$ th instant. Obviously, the dynamic function of the PMA system consists of historic information 


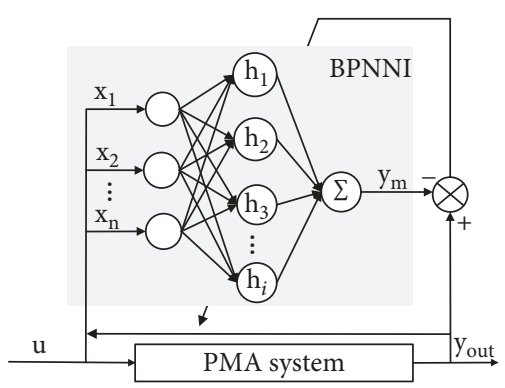

Figure 2: Identification scheme of dynamic model of PMA system based on BP neural network.

of displacements and input voltages and is difficult to analytical description. To reduce the influence of uncertainties on the accuracy of a dynamic model, a BP neural network is employed to approximate the dynamics of PMA system. The scheme is shown in Figure 2. The PMA system comprises a pneumatic muscle actuator, a proportional valve, and an air pipe. The detailed structure of BPNNI is listed in Figure 3.

2.2.2. Identifying Algorithm. The identifying process of dynamics of PMA is tuning weights of BPNN. To avoid divergence when training, different inputs are normalized by following the listed formula:

$$
x_{i}^{\prime}=\frac{x_{i}-x_{\min }}{x_{\max }-x_{\min }}
$$

where $x_{i}^{\prime}$ is the normalized value, $x_{i}$ is the origin value, and $x_{\max }$ and $x_{\min }$ are the maximum value and minimum value of the parametric change interval of $x_{i}$, respectively.

If inputs of the hidden layer are denoted by net ${ }^{(2)}$, where superscript (2) symbols the second layer, i.e., the hidden layer, net ${ }^{(2)}$ is calculated as follows:

$$
\begin{aligned}
\operatorname{net}^{(2)}(k) & =\left[\begin{array}{ccc}
w_{1 \rightarrow 1}^{(2)}(k) & \cdots & w_{m+n+3 \rightarrow l}^{(2)}(k) \\
\vdots & \vdots & \vdots \\
w_{m+n+3 \rightarrow l}^{(2)}(k) & \cdots & w_{m+n+3 \rightarrow l}^{(2)}(k)
\end{array}\right] \cdot X \\
& =\left[\begin{array}{c}
\operatorname{net}_{1}^{(2)}(k) \\
\vdots \\
\operatorname{net}_{l}^{(2)}(k)
\end{array}\right],
\end{aligned}
$$

where $\quad X=[y(k), \cdots, y(k-n), u(k+1), u(k), \cdots, u(k-m)]^{\mathrm{T}}$ is the input vector of BPNNI, net ${ }_{i}^{(2)}$ represents the input of the $i$ th node in the hidden layer, $w_{i \rightarrow j}^{(2)}(i=1 \sim m+n+3$, $j=1 \sim l)$ is defined as the influencing weight of output from the $i$ th node of the input layer on the input of the $j$ th node in the hidden layer.

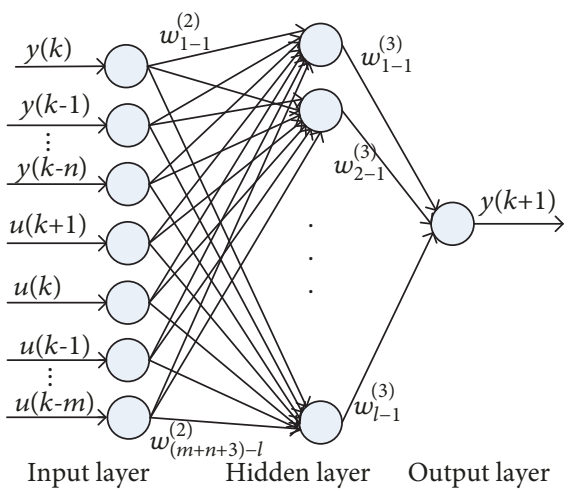

FIgURe 3: BP neural network identifier of PMA system.

$O_{i}^{(2)}(i=1 \sim l)$ is the output of the $i$ th node in the hidden layer. The activation function of the hidden layer is the sigmoid function, represented by $g(x)$. Hence, $O_{i}^{(2)}(i=1 \sim l)$ is calculated as follows:

$$
O_{i}^{(2)}=g\left(\text { net }_{i}^{(2)}\right)=\frac{1}{1+e^{- \text {net }_{i}^{(2)}}} .
$$

The define input of the output layer is net ${ }^{(3)}, w_{i \rightarrow j}^{(3)}(i=$ $1 \sim l, j=1$ ) symbols the influencing weight of the output from the $i$ th node of the hidden layer on the node of the output layer, and $O^{(3)}$ is the output of BPNNI. If the active model of the output layer is the proportional function, we have

$$
y(k+1)=O^{(3)}(k+1)=\operatorname{net}^{(3)}(k)=\sum_{i=1}^{l} w_{i \rightarrow 1}^{(3)}(k) O_{i}^{(2)}(k) .
$$

To minimize errors between the output of BPNNI and sampled values, weights of BPNNI should be adjusted according to some criteria. In this paper, a cost function is defined to provide the criteria as follows:

$$
J(k)=\frac{1}{2}\left(y_{\mathrm{m}}(k+1)-y_{\text {out }}(k+1)\right)^{2} .
$$

Without the loss of generality, the rules of updating weights are obtained in the direction of gradient descent and defined in (5) for the hidden layer and output layer, respectively:

$$
\begin{aligned}
& \Delta w_{i \rightarrow j}^{(2)}(k)=-\eta \frac{\partial J(k)}{\partial w_{i \rightarrow j}^{(2)}(k)}+\alpha \Delta w_{i \rightarrow j}^{(2)}(k-1), \\
& \quad i=1 \sim m+n+3, j=1 \sim l,
\end{aligned}
$$

$$
\Delta w_{i \rightarrow j}^{(3)}(k)=-\eta \frac{\partial J(k)}{\partial w_{i \rightarrow j}^{(3)}(k)}+\alpha \Delta w_{i \rightarrow j}^{(3)}(k-1), \quad i=1 \sim l, j=1,
$$




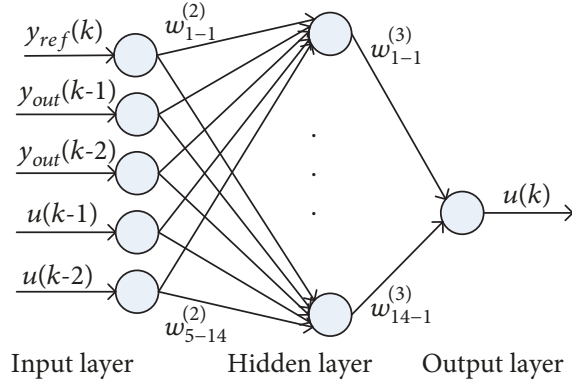

FIGURE 4: BP neural network adaptive controller of PMA system.

where $\eta$ is the learning rate and $\alpha$ is the inertia factor. $(\partial J(k)) /\left(\partial w_{i \rightarrow j}^{(2)}(k)\right)$ and $(\partial J(k)) /\left(\partial w_{i \rightarrow 1}^{(3)}(k)\right)$ are acquired as follows:

$$
\begin{aligned}
\frac{\partial J(k)}{\partial w_{i \rightarrow j}^{(2)}(k)}= & \frac{\partial J(k)}{\partial y_{m}(k)} \cdot \frac{\partial y_{m}(k)}{\partial \operatorname{net}^{(3)}(k)} \cdot \frac{\partial \operatorname{net}^{(3)}(k)}{\partial O_{j}^{(2)}(k)} \\
& \cdot \frac{\partial O_{j}^{(2)}(k)}{\partial \operatorname{net}_{j}^{(2)}(k)} \cdot \frac{\partial \operatorname{net}_{j}^{(2)}(k)}{\partial w_{i \rightarrow j}^{(2)}(k)} \\
= & e_{m}(k) \cdot \frac{\partial f\left(\operatorname{net}_{j}^{(2)}(k)\right)}{\partial \operatorname{net}_{j}^{(2)}(k)} \cdot w_{j \rightarrow 1}^{(3)}(k) \cdot x_{i}^{\prime} \\
\frac{\partial J(k)}{\partial w_{i \rightarrow 1}^{(3)}(k)}= & \frac{\partial J(k)}{\partial y_{m}(k)} \cdot \frac{\partial y_{m}(k)}{\partial \operatorname{net}^{(3)}(k)} \cdot \frac{\partial \operatorname{net}^{(3)}(k)}{\partial w_{i \rightarrow 1}^{(3)}(k)} \\
= & e_{m}(k) \cdot O_{i}^{(2)}(k) .
\end{aligned}
$$

2.3. Adaptive BP Neural Network Controller of PMA System. The BP neural network is employed as the controller of PMA to enhance flexibility and adaptability of the controlling system. Considering the hysteresis and time-varying characteristics of PMA, the input vector of BPNNC is $\left[y_{\text {ref }}\right.$ $\left.(k), y_{\text {out }}(k-1), y_{\text {out }}(k-2), u(k-1), u(k-2)\right]$, the hidden layer owns fourteen nodes, and the output of BPNNC is the controlling signal $u(k)$. The structure of BPNNC is shown in Figure 4. The activation function of the hidden layer also adopts the sigmoid function. Weights of BPNNC are trained online, and the updating rule is gradient descent. The cost function is defined as follows:

$$
\Theta(k)=\frac{1}{2}\left(y_{r e f}(k+1)-y_{m}(k+1)\right)^{2} .
$$

Updating formulas of weights in the hidden layer and output layer are listed as follows in (12) and (13), respectively:

$$
\begin{aligned}
& \Delta w_{i \rightarrow j}^{(2)}(k)=-\varphi \frac{\partial \Theta(k)}{\partial w_{i \rightarrow j}^{(2)}(k)}+\gamma \Delta w_{i \rightarrow j}^{(2)}(k-1), \\
& \quad i=1 \sim m+n+3, j=1 \sim l,
\end{aligned}
$$

$$
\Delta w_{i \rightarrow j}^{(3)}(k)=-\varphi \frac{\partial \Theta(k)}{\partial w_{i \rightarrow j}^{(3)}(k)}+\gamma \Delta w_{i \rightarrow j}^{(3)}(k-1), \quad i=1 \sim l, j=1,
$$

where $\varphi$ is the learning rate and $\gamma$ is the inertia factor. $(\partial \Theta(k)) /\left(\partial w_{i \rightarrow j}^{(2)}(k)\right)$ and $(\partial \Theta(k)) /\left(\partial w_{i \rightarrow 1}^{(3)}(k)\right)$ are acquired as follows:

$$
\begin{aligned}
\frac{\partial \Theta(k)}{\partial w_{i \rightarrow j}^{(2)}(k)}= & \frac{\partial \Theta(k)}{\partial y_{m}(k)} \cdot \frac{\partial y_{m}(k)}{\partial u(k)} \cdot \frac{\partial u(k)}{\partial \operatorname{net}^{(3)}(k)} \\
& \cdot \frac{\partial \operatorname{net}^{(3)}(k)}{\partial O_{j}^{(2)}(k)} \cdot \frac{\partial O_{j}^{(2)}(k)}{\partial \operatorname{net}_{j}^{(2)}(k)} \cdot \frac{\partial \operatorname{net}_{j}^{(2)}(k)}{\partial w_{i \rightarrow j}^{(2)}(k)} \\
= & -e_{c} \cdot \frac{\partial y_{m}(k)}{\partial u(k)} \cdot w_{j \rightarrow 1}^{(3)}(k) \cdot \frac{\partial f\left(\operatorname{net}_{j}^{(2)}(k)\right)}{\partial \operatorname{net}_{j}^{(2)}(k)} \cdot x_{i}, \\
\frac{\partial \Theta(k)}{\partial w_{i \rightarrow 1}^{(3)}(k)}= & \frac{\partial \Theta(k)}{\partial y_{m}(k)} \cdot \frac{\partial y_{m}(k)}{\partial u(k)} \cdot \frac{\partial u(k)}{\partial \operatorname{net}^{(3)}(k)} \cdot \frac{\partial \operatorname{net}^{(3)}(k)}{\partial w_{i \rightarrow j}^{(2)}(k)} \\
= & -e_{c} \cdot \frac{\partial y_{m}(k)}{\partial u(k)} \cdot O_{i}^{(2)}(k) .
\end{aligned}
$$$$
\partial y_{m}(k) / \partial u(k) \text { is acquired from BPNNI: }
$$

$$
\frac{\partial y_{m}(k)}{\partial u(k)}=\sum_{i=1}^{19} w_{i \rightarrow 1}^{(3)}(k) \frac{\partial f\left[\operatorname{net}_{i}^{(2)}(k)\right]}{\partial \operatorname{net}_{i}^{(2)}(k)} w_{4 \rightarrow i}^{(2)}(k) .
$$

\section{Experiments and Discussions}

3.1. Approximation of PMA System by NNI. A single PMA mass system set-up is presented in Figure 5 to validate the NNI method and NNC algorithm. PMA is FESTO DMSP20-180N-RM-CM with an inner diameter of $20 \mathrm{~mm}$ and an initial length of $180 \mathrm{~mm}$. The pressure range of PMA is $[0,0.6] \mathrm{MPa}$, and the maximum theoretical contractile ratio is $25 \%$ of the nominal length. The weight of the mass is $4.25 \mathrm{~kg}$. Controlling valve is SMC ITV1050-212N with an output pressure range of [0.005 0.9] $\mathrm{MPa}$ and an output voltage range of $[0,5] \mathrm{V}$. The relationship between output voltage and output pressure of ITV1050-212N is linear. AD/DA card is USB3102A of USB Data Acquisition Device from ART Technology. The device owns sixteen analogue sampling channels with an accuracy of 16-bit and speed of $250 \mathrm{kS} / \mathrm{s}$ and supports single-point sampling mode, multiple-point sampling mode, and continuous sampling mode. Besides, this card owns two DA channels and twelve I/O pins. The air compressor supplies air to the system, and the valve regulates the air accurately according to commands from the host computer. The draw-wire displacement sensor is attached to the mass and measures real-time displacement of PMA. The computer is responsible for human-computer interaction, approximates the dynamics of PMA system, and performs the adaptive neural network algorithm. 


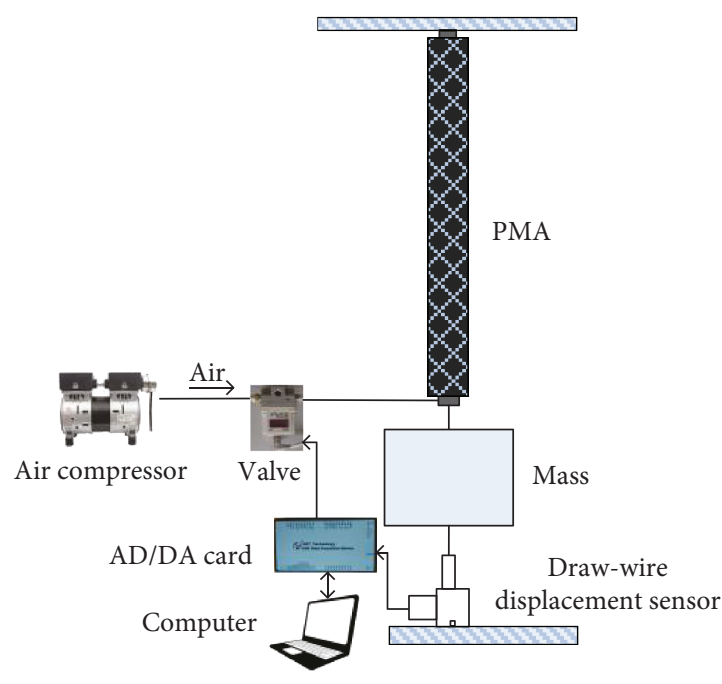

(a) Schematic drawing

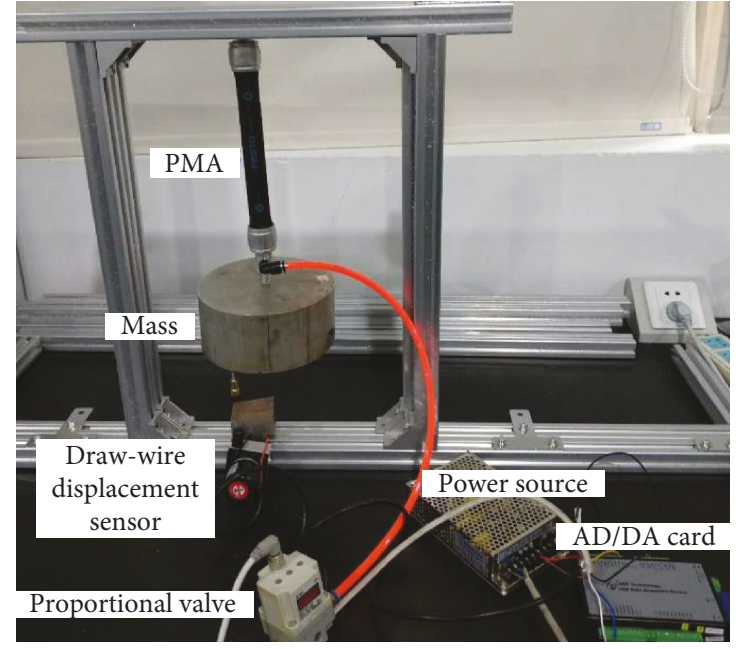

(b) Photograph of the experimental set-up

Figure 5: The single PMA mass system.

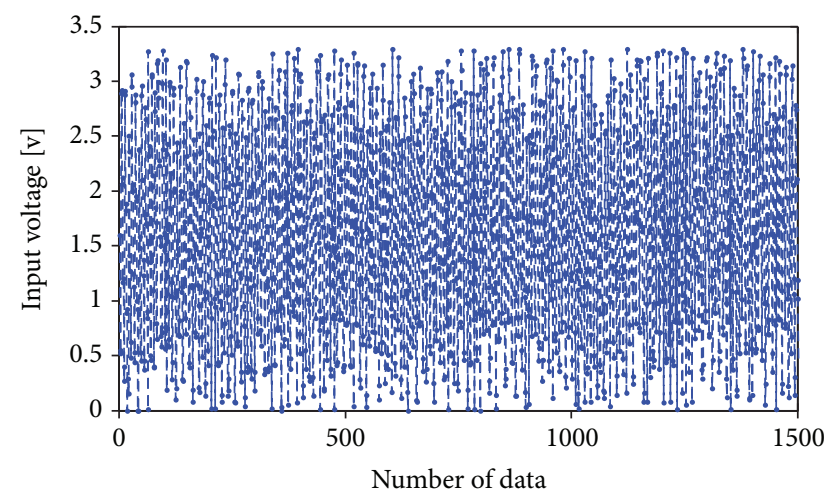

FIGURE 6: Input voltage of valve in random forms for NNI.

3.1.1. Offline Model of PMA System. In this paper, the NNI model is built up by offline data firstly, and then online modification is performed to improve model accuracy. $[y(k), y(k-1), y(k-2), u(k+1), u(k), u(k-1), u(k-2)]$ is selected as the input vector of the neural network identifier. $y(k)$ and $u(k)$ are the displacement of PMA and the input voltage of proportional valve, respectively, at the $k$ th sampling instant. $y(k-1)$ and $y(k-2)$ are selected as the input elements of NNI, which signifies the historic velocity and acceleration of PMA which are taken into account.

To obtain experimental data from the apparatus, controlling voltage in random wave forms with value distributing throughout the interval $[0,3.3] \mathrm{V}$ is applied to the PMA system. The controlling voltage is shown in Figure 6. The corresponding output displacement of the PMA system is shown in Figure 7. Command time between neighbouring inputs in the identification experiment is $0.5 \mathrm{~s} .1500$ points are sampled for offline model identifying, and 200 points are used to validate the established neural network model.

By trials and errors, a number of nodes of each layer are determined. The input layer, single hidden layer, and output layer of BPNNI own seven nodes (i.e., $m=2$ and $n=2$ in

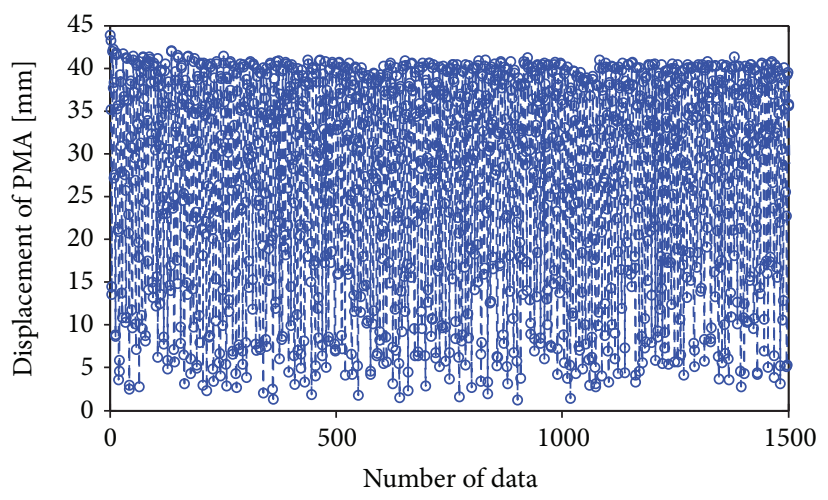

Figure 7: Displacement of PMA system under the random input voltages.

Figure 3), twelve nodes (i.e., $l=12$ in Figure 3), and one node, respectively. The comparison between predicted displacements of established NNI of PMA system and actual values is shown in Figure 8. Predicting errors of NNI of PMA system are plotted in Figure 9. The maximum predicting error is $3.7187 \mathrm{~mm}$, and the standard deviation is $1.327 \mathrm{~mm}$. Figure 10 shows the predicting error rate of NNI of PMA system, where the maximum error rate is $10 \%$, the minimum error rate is $1.78 \%$, the and standard deviation is $6.04 \%$. The aforementioned accuracy indicates good depicting capacity of the dynamics of PMA system.

3.1.2. Online NNI Model of PMA System. PMA is a timevarying and highly nonlinear system due to its inherent structure and pneumatic driving approach. Hence, online regulation of the established offline NNI model should be performed in order to make further improvement of model accuracy. The adjusting criterion of the neural network identifier is shown in (6). Weights of the hidden and output nodes are regulated by obeying (7), (8), (9), and (10). The online NNI model is to supply gradient descent information to the neural network controller of the PMA system. 


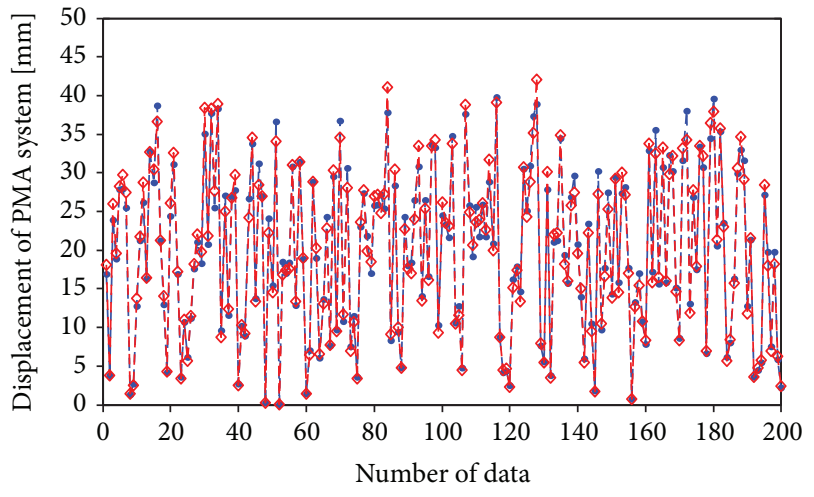

- Actual displacements of PMA system

$\diamond$ Predicted displacements of NNI of PMA system

FIGURE 8: Predicted values of NNI and actual displacements of PMA system.

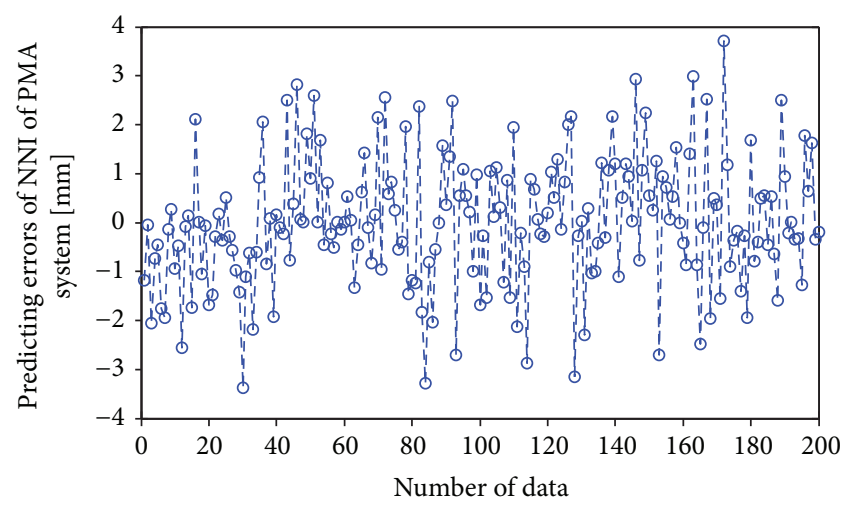

FIGURE 9: Errors of predicted model and actual system.

3.2. Trajectories Tracking Experiments Based on BPNNI and $B P N N C$. On the basis of the presented BPNNI model of PMA system in Section 3.1, the back propagation neural network controller (BPNNC) is employed to realize accurately position tracking. Firstly, lots of training practice are done to regulate initial values of weights until the performance of BPNNC is satisfactory. To validate the actual performance of BPNNC, square wave excitation and sinusoidal trajectory motions with different frequencies and amplitudes are employed to drive the PMA system. A classic PID algorithm is also adapted to control the PMA system, and the comparison between performances of BPNNC and classic PID is done. Square wave excitation with a frequency of $0.1 \mathrm{~Hz}$ and an amplitude of $10 \mathrm{~mm}$ is applied to the PMA system, and tracking results are shown in Figure 11. The rising time of the adaptive BPNNI-BPNNC response and that of the PID response at time interval $[0,5] \mathrm{s}$ are $0.5 \mathrm{~s}$ and $0.67 \mathrm{~s}$, respectively. At the descend stage, the steady error of the proposed adaptive controller is much smaller than that of the PID controller. Position tracking error curves of the two controllers are compared in Figure 12. Obviously, adaptive BPNNI-BPNNC shows higher accuracy than classic PID. Figure 13 is the enlarged view of responses at time interval $[10,15]$ s. It can be indicated that the performance of NNI-

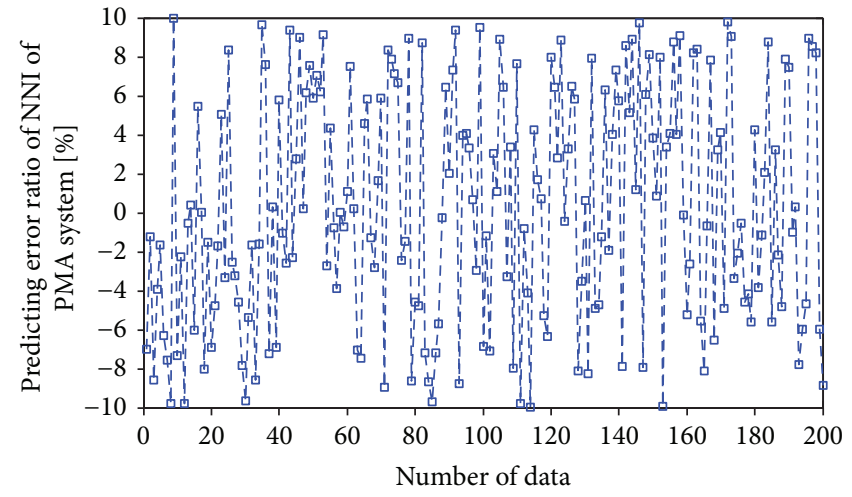

FIgURe 10: Predicting error rate of NNI of PMA system.

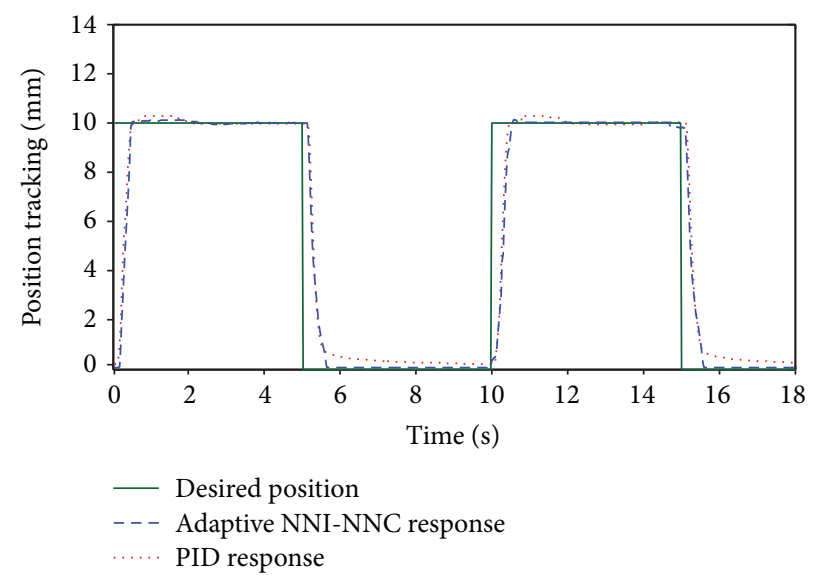

FIgURE 11: Tracking performances of two controllers under square waves of $0.1 \mathrm{~Hz}$ with an amplitude of $10 \mathrm{~mm}$.

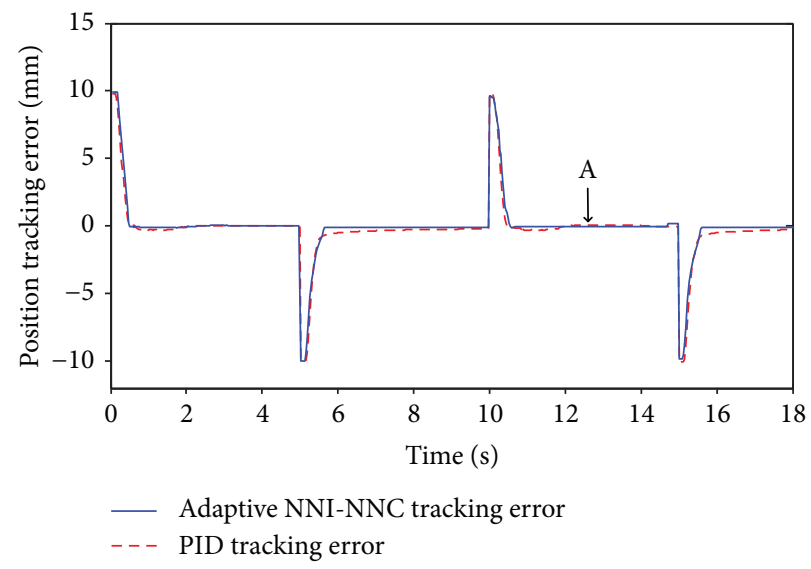

FIGURE 12: Tracking errors of two controllers under square waves of $0.1 \mathrm{~Hz}$ with an amplitude of $10 \mathrm{~mm}$.

NNC is much steadier and less oscillation occurs in the response of adaptive BPNNI-BPNNC.

Square wave excitation with a frequency of $0.2 \mathrm{~Hz}$ and an amplitude of $18 \mathrm{~mm}$ is used as the referred trajectory of the PMA system. Outputs of the adaptive BPNNI-BPNNC system and classic PID controller are drawn in Figure 14. The maximum overshoots of the adaptive BPNNI-BPNNC 


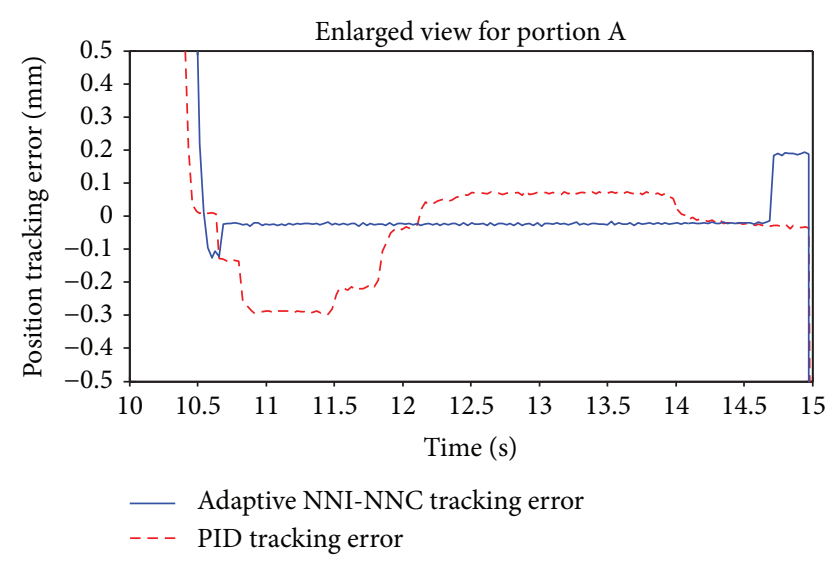

Figure 13: Enlarged view of tracking errors.

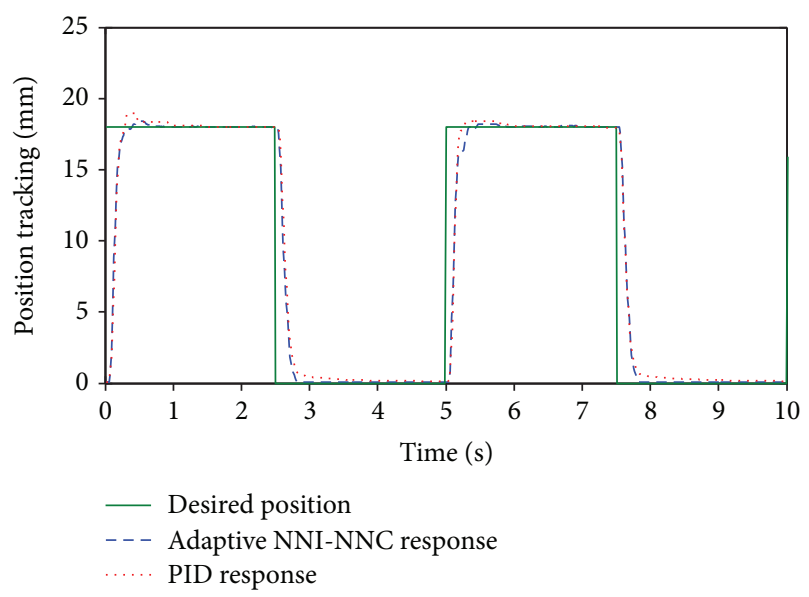

FIGURE 14: Tracking performances of two controllers under square waves of $0.2 \mathrm{~Hz}$ with an amplitude of $18 \mathrm{~mm}$.

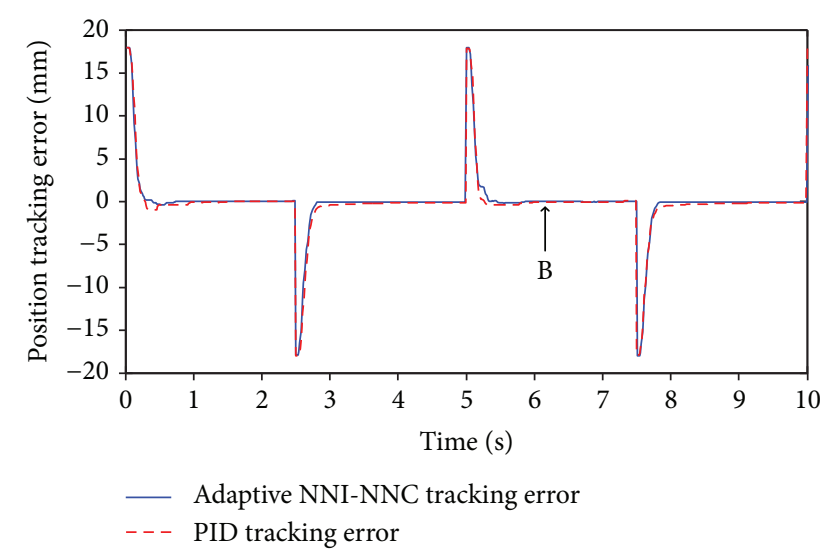

FIGURE 15: Tracking errors of two controllers under square waves of $0.2 \mathrm{~Hz}$ with an amplitude of $18 \mathrm{~mm}$.

response and classic PID response are 3.02\% and 5.39\%, respectively. The steady tracking performance of the BPNNI-BPNNC response is also better than that of the classic PID controller by observing Figures 15 and 16.

To further validate the capacity of the proposed adaptive controller, sinusoidal trajectory motion with a frequency of

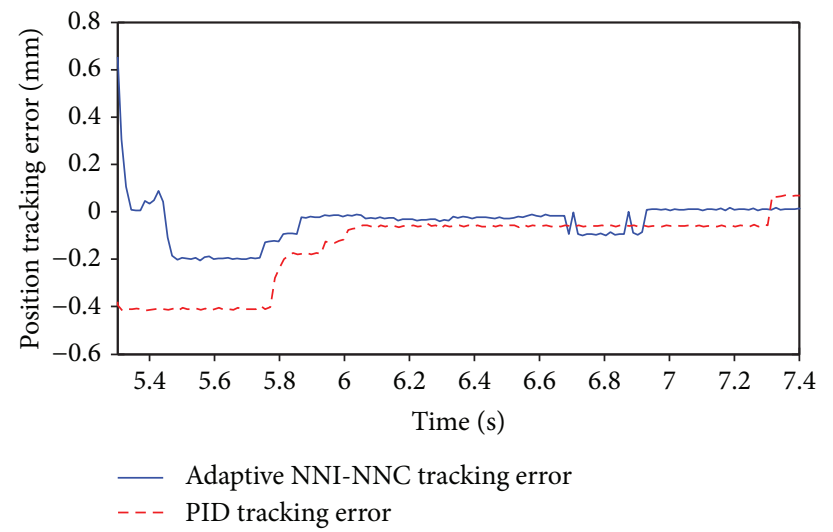

FIGURE 16: Enlarged view of tracking errors.

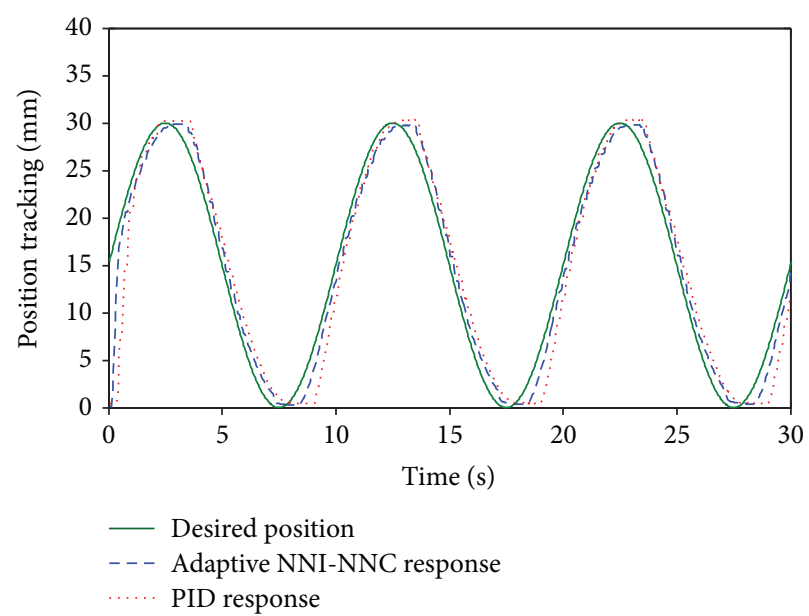

FIgURE 17: Tracking performances of two controllers under sinusoidal waves of $0.1 \mathrm{~Hz}$ with an amplitude of $30 \mathrm{~mm}$.

$0.1 \mathrm{~Hz}$ and an amplitude of $30 \mathrm{~mm}$ is applied to the PMA system. Performances of BPNNI-BPNNC response and classic PID response are plotted in Figure 17. Obviously, the tracking accuracy of the adaptive controller is higher than that of a classic PID controller. The response speed of BPNNI$\mathrm{BPNNC}$ is also higher than that of the classic PID controller.

\section{Conclusion}

The PMA system owns high nonlinearities due to its inherent structure and pneumatic driving characteristics, which make it difficult to realize accurate position control. This paper adopts the BP neural network to build up an accurate identifier to model the dynamics of a PMA-driven setup. The BPNNI adopts historic displacements, corresponding historic controlling voltages and desired voltage command as the input vector, and produces desired displacement as the output of the neural network. On the basis of the proposed BPNNI, a BP neural network controller is designed to control position tracking. The proposed BPNNI is tuned online and provides the gradient descent information for BPNNC. Experiments are performed to evaluate the adaptive BPNNI-BPNNC controlling system, and the results demonstrate the attractive capacity of the novel controller. 


\section{Data Availability}

The datasets used or analysed during the current study are available from the corresponding author on reasonable request.

\section{Conflicts of Interest}

The authors declare that there is no conflict of interests regarding the publication of this paper.

\section{Acknowledgments}

This work is supported by the Natural Science Foundation of Jiangsu Province (BK20170301), Fundamental Research Funds for the Central Universities (2016B11814), Science and Technology Planning Projects of Changzhou (CJ20179042), Jiangsu Province Key Development Program (BE2017647), and Projects of International Cooperation and Exchanges of Changzhou (CZ20170018).

\section{References}

[1] T. Kaneko, M. Sekiya, K. Ogata, S. Sakaino, and T. Tsuji, "Force control of a jumping musculoskeletal robot with pneumatic artificial muscles," in 2016 IEEE/RSJ International Conference on Intelligent Robots and Systems (IROS), pp. 5813-5818, Daejeon, Republic of Korea, 2016, IEEE.

[2] W. He, L. Kong, Y. Dong, Y. Yu, C. Yang, and C. Sun, "Fuzzy tracking control for a class of uncertain MIMO nonlinear systems with state constraints," IEEE Transactions on Systems, Man, and Cybernetics: Systems, no. 99, pp. 1-12, 2017.

[3] T. Deaconescu and A. Deaconescu, "Pneumatic muscle actuated isokinetic equipment for the rehabilitation of patients with disabilities of the bearing joints," in Proceedings of the International MultiConference of Engineers and Computer Scientists 2009 (IMECS 2009), vol. 2, pp. 1823-1827, Kowloon, Hong Kong, 2009.

[4] X. Li, H. Xia, and T. Guan, "Development of legs rehabilitation exercise system driven by pneumatic muscle actuator," in 2008 2nd International Conference on Bioinformatics and Biomedical Engineering, pp. 1309-1311, Shanghai, China, 2008, IEEE.

[5] G. Tao, X. Zhu, B. Yao, and J. Cao, “Adaptive robust posture control of a pneumatic muscles driven parallel manipulator with redundancy," in 2007 American Control Conference, pp. 764-769, New York, NY, USA, 2009.

[6] H. Aschemann and D. Schindele, "Sliding-mode control of a high-speed linear axis driven by pneumatic muscle actuators," IEEE Transactions on Industrial Electronics, vol. 55, no. 11, pp. 3855-3864, 2008.

[7] S. Q. Xie and P. K. Jamwal, "An iterative fuzzy controller for pneumatic muscle driven rehabilitation robot," Expert Systems with Applications, vol. 38, no. 7, pp. 8128-8137, 2011.

[8] T. Y. Choi, B. S. Choi, and K. H. Seo, "Position and compliance control of a pneumatic muscle actuated manipulator for enhanced safety," IEEE Transactions on Control Systems Technology, vol. 19, no. 4, pp. 832-842, 2011.

[9] C. Yang, Y. Jiang, W. He, J. Na, Z. Li, and B. Xu, “Adaptive parameter estimation and control design for robot manipulators with finite-time convergence," IEEE Transactions on Industrial Electronics, vol. 65, no. 10, pp. 8112-8123, 2018.
[10] S. Balasubramanian, H. Huang, and J. He, "Quantification of dynamic property of pneumatic muscle actuator for design of therapeutic robot control," in 2006 International Conference of the IEEE Engineering in Medicine and Biology Society, pp. 2734-2737, New York, NY, USA, 2016, IEEE.

[11] J. Sarosi, I. Biro, J. Nemeth, and L. Cveticanin, "Dynamic modeling of a pneumatic muscle actuator with two-direction motion," Mechanism and Machine Theory, vol. 85, pp. 2534, 2015.

[12] M. K. Chang, "An adaptive self-organizing fuzzy sliding mode controller for a 2-DOF rehabilitation robot actuated by pneumatic muscle actuators," Control Engineering Practice, vol. 18, no. 1, pp. 13-22, 2010.

[13] R. Amar, H. Mustapha, and T. Mohamed, "Decentralized RBFNN type-2 fuzzy sliding mode controller for robot manipulator driven by artificial muscles," International Journal of Advanced Robotic Systems, vol. 9, no. 5, p. 182, 2012.

[14] G. Andrikopoulos, G. Nikolakopoulos, and S. Manesis, "Advanced nonlinear PID-based antagonistic control for pneumatic muscle actuators," IEEE Transactions on Industrial Electronics, vol. 61, no. 12, pp. 6926-6937, 2014.

[15] J. Wu, J. Huang, Y. Wang, and K. Xing, "Nonlinear disturbance observer-based dynamic surface control for trajectory tracking of pneumatic muscle system," IEEE Transactions on Control Systems Technology, vol. 22, no. 2, pp. 440455, 2014.

[16] V. T. Jouppila, S. A. Gadsden, G. M. Bone, A. U. Ellman, and S. R. Habibi, "Sliding mode control of a pneumatic muscle actuator system with a PWM strategy," International Journal of Fluid Power, vol. 15, no. 1, pp. 19-31, 2014.

[17] C. J. Lin, C. R. Lin, S. K. Yu, and C. T. Chen, "Hysteresis modeling and tracking control for a dual pneumatic artificial muscle system using Prandtl-Ishlinskii model," Mechatronics, vol. 28, pp. 35-45, 2015.

[18] G. Andrikopoulos, G. Nikolakopoulos, I. Arvanitakis, and S. Manesis, "Switching model predictive control of a pneumatic artificial muscle," International Journal of Control, Automation and Systems, vol. 11, no. 6, pp. 1223-1231, 2013.

[19] D. X. Ba, T. Q. Dinh, and K. K. Ahn, "An integrated intelligent nonlinear control method for a pneumatic artificial muscle," IEEE/ASME Transactions on Mechatronics, vol. 21, no. 4, pp. 1835-1845, 2016.

[20] C. Yang, X. Wang, L. Cheng, and H. Ma, "Neural-learningbased telerobot control with guaranteed performance," IEEE Transactions on Cybernetics, vol. 47, no. 10, pp. 3148-3159, 2017.

[21] B. P. M. Beneš, Analysis of Industrial Hoist Mechanisms Braking Torque and Deceleration Control with Neural Networks, [M.S. thesis], Czech Technical University in Prague, 2014.

[22] Y. Zhang and X. Zhu, "Medical image study based on CPSOBP neural network," International Journal of Digital Content Technology and its Applications, vol. 6, no. 19, pp. 297-304, 2012.

[23] X. Tu, Y. F. Zhou, P. Zhao, and X. Cheng, "Modeling the static friction in a robot joint by genetically optimized BP neural network," Journal of Intelligent \& Robotic Systems, pp. 1-13, 2018.

[24] Y. Cai, Q. Zhan, X. Xi, and A. Rahmani, "Inverse kinematics identification of a spherical robot based on BP neural networks," in 2011 6th IEEE Conference on Industrial Electronics and Applications, pp. 2114-2119, Beijing, China, 2011, IEEE. 
[25] C. Yang, Y. Jiang, Z. Li, W. He, and C. Y. Su, "Neural control of bimanual robots with guaranteed global stability and motion precision," IEEE Transactions on Industrial Informatics, vol. 13, no. 3, pp. 1162-1171, 2017.

[26] T. Vo-Minh, T. Tjahjowidodo, H. Ramon, and H. van Brussel, "A new approach to modeling hysteresis in a pneumatic artificial muscle using the Maxwell-slip model," IEEE/ASME Transactions on Mechatronics, vol. 16, no. 1, pp. 177-186, 2011. 


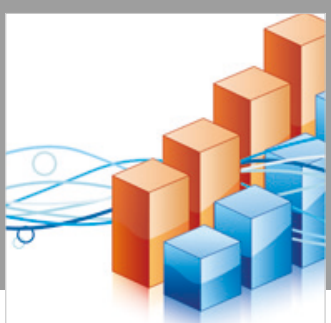

Advances in

Operations Research

\section{-n-m}
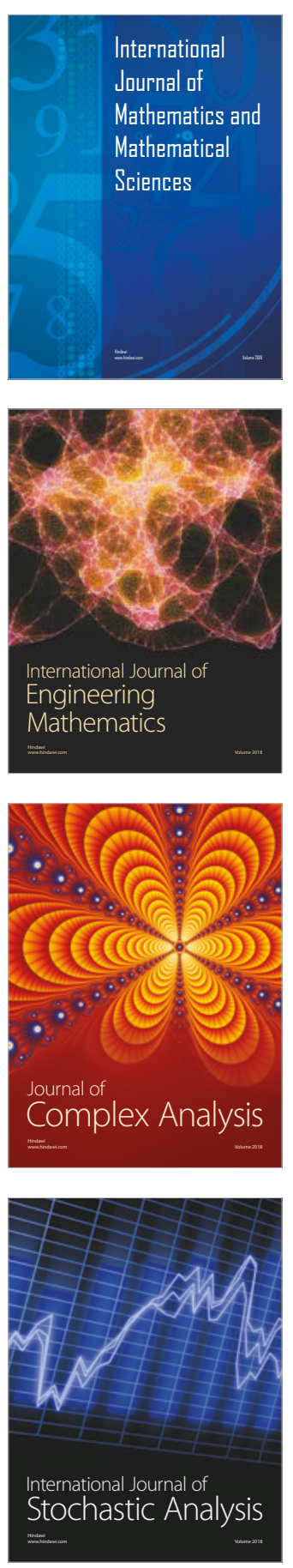
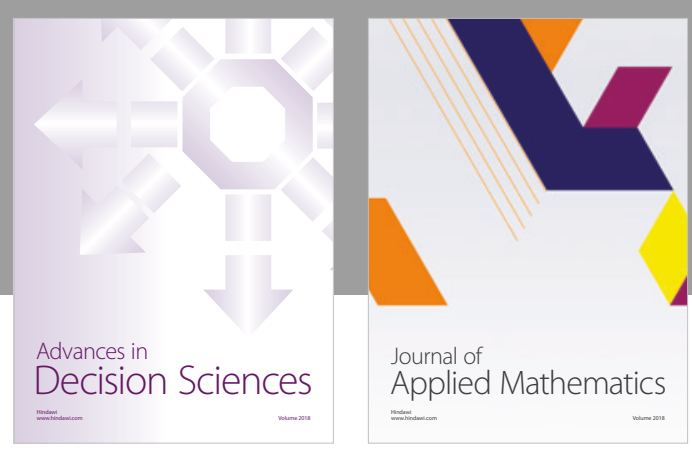

Journal of

Applied Mathematics
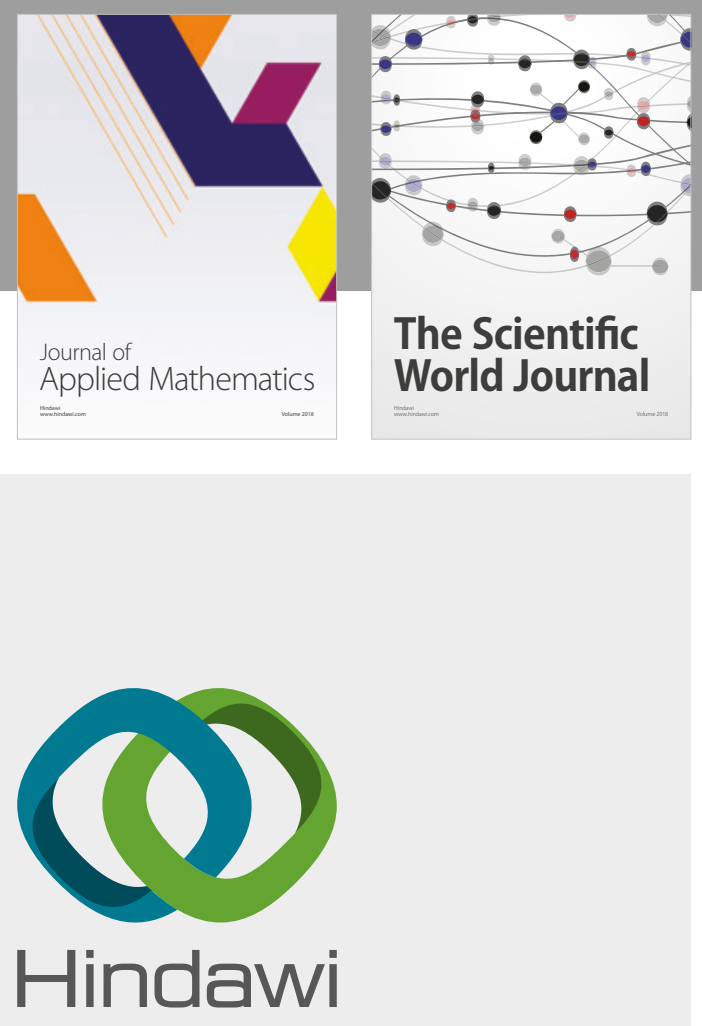

Submit your manuscripts at

www.hindawi.com

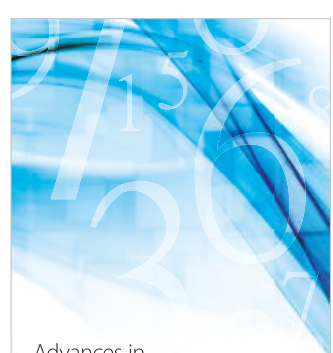

Advances in
Numerical Analysis
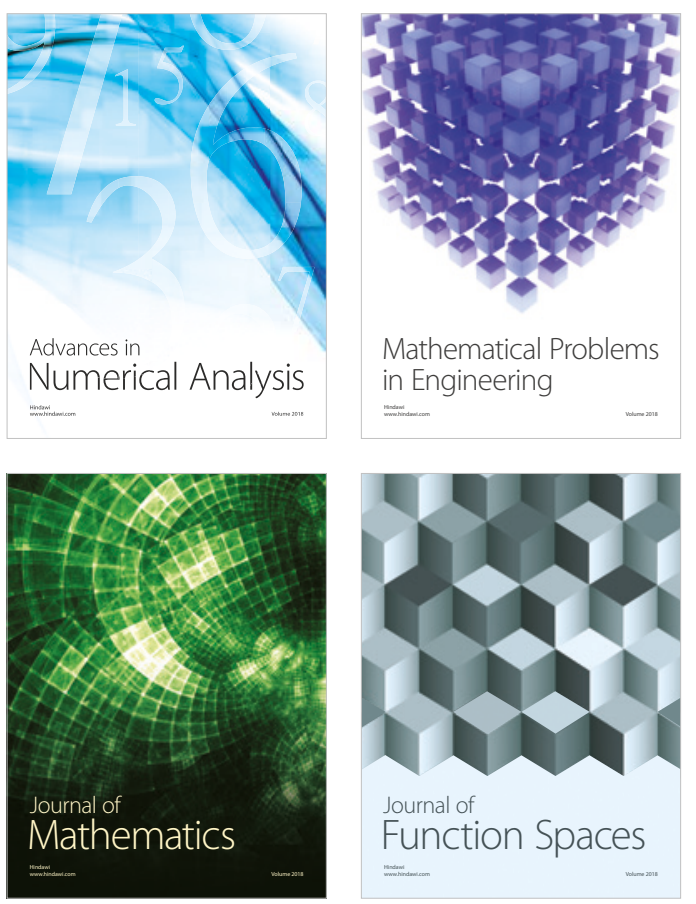

Mathematical Problems in Engineering

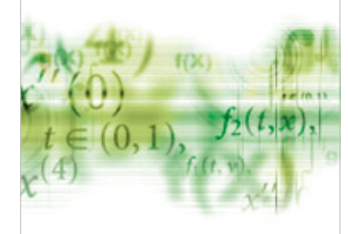

International Journal of

Differential Equations

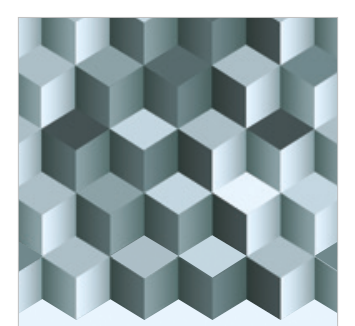

Journal of

Function Spaces
The Scientific

World Journal

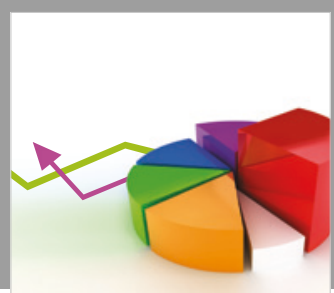

Journal of

Probability and Statistics
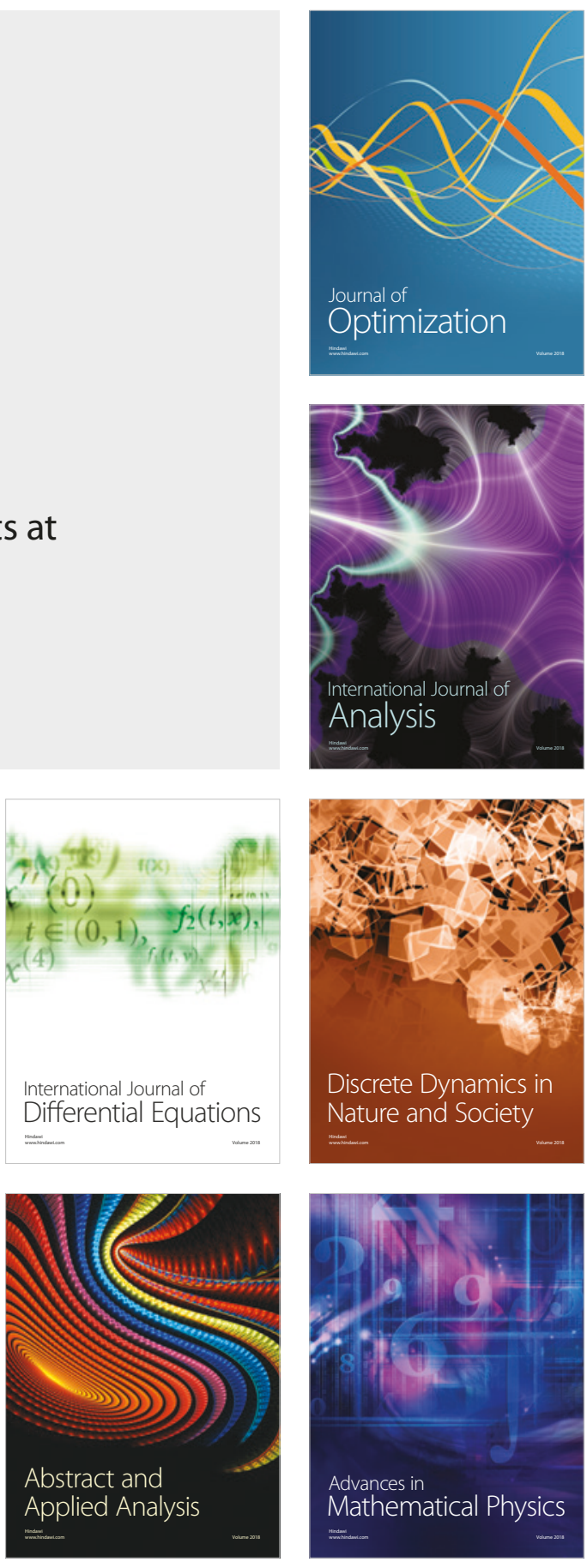